\title{
Molecular Genetics of Familial Alzheimer's Disease
}

\author{
P.H. St. George-Hyslop, J.L. Haines, R.J. Polinsky, R.E. Tanzi, L. Farrer, \\ R.H. Myers and J.F. Gusella
}

\begin{abstract}
The molecular genetic strategies aimed at isolating and characterizing the defective gene(s) in familial Alzheimer's disease, the inherent limitations of the techniques and recent progress in the field are reviewed. Three independent groups have found an apparent linkage to chromosome 21 but two other groups have not, suggesting that familial Alzheimer's disease may be etiologically heterogeneous.

RÉSUMÉ: Génétique moléculaire de la maladie d'Alzheimer familiale Nous revoyons les stratégies utilisées en génétique moléculaire pour isoler et caractériser le(s) gène(s) anormal(aux) dans la maladie d'Alzheimer familiale, les limitations inhérentes à ces techniques et les progrès récents dans ce domaine. Trois groupes indépendants de chercheurs ont observé une liaison au chromosome 21, alors que deux autres groupes n'ont par retrouvé cette liaison, suggérant que l'etiologie de la maladie d'Alzheimer familiale peut être hétérogène.
\end{abstract}

Can. J. Neurol. Sci. 1989; 16: 465-467

The etiology of Alzheimer's Disease [AD] remains unknown, indeed it is uncertain whether this disease represents a single entity, or a syndrome composed of multiple illnesses of different etiologies.but with similar phenotypes. Recent advances in molecular biology and molecular genetics may provide tools to address some of these questions.

Recently, several epidemiological surveys have suggested that first degree relatives of patients with Alzheimer's disease have an age adjusted risk of developing this disorder which approximates the $50 \%$ risk ratio expected for an autosomal dominant trait. ${ }^{1}$ These observations, together with the description of increasing numbers of pedigrees with reasonably clear autosomal dominant transmission of the Alzheimer phenotype, have led to speculation that $\mathrm{AD}$ may represent a very common genetic disorder transmitted as an autosomal dominant trait with age dependent penetrance. Whether the autosomal dominant transmission of AD observed in large pedigrees such as the " $N$ " pedigree of Foncin et $\mathrm{al}^{2}$ can ultimately be generalized to all instances of AD remains to be determined. However, the existence of such pedigrees provides a basis for attempts to isolate and characterize the defective Familial Alzheimer's Disease [FAD] gene using modern molecular genetic strategies. Even if the autosomal dominant and the sporadic forms of AD ultimately prove to be different disorders, knowledge of the pathogenesis of FAD afforded by these studies may provide useful insights into possible mechanisms causing sporadic AD. The molecular genetic strategies, their inherent limitations, and recent progress achieved using these strategies will be discussed.

\section{Introduction to Molecular Genetic Methods}

Theoretically, a disease gene whose identity is not known can be isolated and characterized using the following sequential paradigm. The first step towards cloning of the disease gene is to determine which chromosome carries the disease mutation. The chromosomal localization of a disease gene can be defined by employing genetic linkage studies to discover genetic markers [e.g. RFLPs] which co-segregate with the disease phenotype in large pedigrees showing the disorder in question. Observation of such co-segregation or genetic linkage, implies that the disease gene and the genetic marker are located close together on the same chromosome. Since the chromosomal location of the genetic marker is already known, the chromosomal location of the disease gene can thus be deduced. Subsequently, other strategies must be employed in order to isolate the normal and defective alleles from the "linked" chromosomal locus. These "second phase" strategies include chromosome walking, screening cDNA libraries for genes displaying differential expression between affected and control tissues, and long range physical mapping of normal and disease chromosomes using pulse field gel electrophoresis [PFGE] to reveal small interstitial chromosomal rearrangements [e.g. deletions or duplications] which may represent the site of actual mutation.

From the Molecular Neurogenetics Laboratory, Research 3, Massachusetts General Hospital, Boston (PHSG-H, JLH, RET and JFG), the Neurogenetics Laboratory and School of Medicine, Boston University School of Medicine, Boston (LF and RHM), and the Neuropharmacology Division, Medical Neurology Branch, NINCDS, Bethesda (RJP)

Reprint request to: P.H. St. George-Hyslop, Molecular Neurogenetics Lab, Research 3, Massachusetts General Hospital, 32 Fruit St., Boston MA, U.S.A. 02114 


\section{Limitations of The Method in FAD}

Several factors limit the ability of this approach to define successfully a very precise chromosomal location for the FAD gene. ${ }^{3}$ First, the late onset of symptoms and short life expectancy of affected individuals results in pedigrees with relatively few living affected individuals available for study at any given time. As a result, most FAD pedigrees do not provide a powerful substrate for genetic linkage studies. Indeed, simulation studies have shown that in many small nuclear families with the pedigree structure typical of late onset disorders such as FAD, it may be impossible to detect linkage between the disease gene and a genetic marker when they are separated by more than a few million nucleotides. Second, the statistical analysis of the genetic data generated from family studies of any genetic disease requires the inclusion of a number of additional parameters such as: the frequency of the disease gene in the general population [to assess the possibility that the disease was acquired from the "married-in" parent]; estimates of "age-dependent" penetrance and "overall" penetrance of the disease gene [to assess data contributed by currently normal pedigree members who may be pre-symptomatic gene carriers]; estimates of the incidence of new mutations and non-genetic phenocopies [to assess the possibility that some affected members of the pedigree may represent phenocopies or new mutations rather than genetic transmission]. Unfortunately, none of these parameters are known for FAD with precision.

\section{Current Molecular Genetic Results}

We have previously reported positive lod scores in FAD for two anonymous DNA markers located on the proximal portion of chromosome 21 [2 point scores: D21S1/D21S1] $\hat{Z}=2.3$ at $\hat{\theta}=0.08$; D21S IS $16 \hat{Z}=2.3$ at $\hat{\theta}=0.00$; multipoint peak score $\hat{\mathrm{Z}}=4.31 .{ }^{4}$ This suggested that a defective gene causing FAD at least in those degrees we examined may be located on the proximal portion of the long arm of chromosome 21 . We subsequently provided evidence that this genetic defect was not located within the coding sequences of the beta amyloid gene. 5 These results have now been replicated for the pedigree FAD4 by an independent group, ${ }^{6}$ and have been confirmed in additional pedigrees by two other groups. Goate et $\mathrm{al}^{7}$ have shown a multipoint score of 3.4 at a location centromeric to D21S1/D21S11 close to D21S16, while Van Broeckhoven et al ${ }^{8}$ have reported positive scores $[\hat{\mathrm{Z}}=1.5$ at $\hat{\theta}=0.09]$ in two additional families with a marker D21S13 which is also located centromeric to the marker D21S1S11. However, two other groups 9,10 have reported negative results, at least with the D21S1/D21S11 locus, which has led to the suggestion of non-allelic genetic heterogeneity in FAD. It may be important to note, however, that Roses et al ${ }^{9}$ have recently reported positive scores for the D21S16 locus $[\hat{Z}=1.3$ at $\hat{\theta}=0.00]$.

\section{Current Genetic Epidemiology Results}

Familial aggregation of $A D$ has been reported by several authors. However, as indicated earlier, such familial aggregation does not necessarily prove the presence of a genetic defect. Three general epidemiologic strategies have been applied in an attempt to discriminate between familial aggregation due to inheritance and familial aggregation due to shared environment or mixed etiologies. First, segregation studies have been performed in specific pedigrees with multiple affected members in order to discover whether the segregation of the disease in a family conforms to known patterns of mendelian inheritance. Second, population studies can be performed to examine the risk of disease in first degree relatives of patients, as opposed to the risk of disease in first degree relatives of controls. Estimates of the cumulative risk in siblings and in parents of affected individuals may suggest a particular mode of transmission (genetic vs. non-genetic) and a particular type of inheritance in genetic cases (dominant, recessive, sex-linked). Finally, differences in rates of concordance for the disease between monozygotic and dizygotic twins may reveal the relative importance of genetic and environmental agents.

Many of these epidemiological tools have been applied to the study of genetic and environmental causes of AD. Unfortunately, these studies have not produced unequivocal results. Several population studies and family studies have implied that a significant proportion of pre-senile AD may be transmitted as an autosomal dominant trait. However, the data supporting a similar form of inheritance in late onset cases is less clear. Breitner and colleagues' have observed that the cumulative risk for $\mathrm{AD}$ in relatives of both early and late onset AD approximates the $50 \%$ risk ratio expected for an autosomal dominant disease. An increased risk of $A D$ in relatives of patients with late onset $\mathrm{AD}$ has also been reported by Heyman et al 11 and by Farrer et al. ${ }^{12}$ However, in both of the latter studies of late onset $A D$, the observed increase in risk to first degree relatives did not conform to the segregation ratios expected for an autosomal dominant trait. Similarly, in a case controlled study, Chandra et al ${ }^{13}$ found no increased risk of dementia in relatives of patients with late onset, clinically diagnosed $A D$. Studies of twins have been limited by small sample sizes, by retrospective design, and by absence of pathological confirmation of $\mathrm{AD}$. Nee et al 14 have reported that $14 / 32$ monozygotic twins were concordant for $\mathrm{AD}$, while $3 / 7$ dizygotic twins were concordant. With the caveats noted above, the low concordance rates for $\mathrm{AD}$ amongst monozygotic twins, which are approximately similar to those of non-identical dizygotic twins, does not provide unarguable evidence in favour of a genetic defect as the only cause of AD.

\section{Future Directions}

To pursue the hypothesis of a genetic defect on chromosome 21 and to examine the possible existence of other disease loci on other chromosomes we have pursued three strategies.

First, we have concentrated our efforts upon gathering several additional pedigrees which display: 1] clinical and pathological evidence of $A D ; 2$ ] strong evidence for autosomal dominant transmission; and 3] sufficient numbers of living affected individuals within a pedigree structure which would provide an efficient substrate for genetic linkage studies. It is important to note that no attempt has been made to select pedigrees by age of onset, by particular ethnic group, or by other criteria which may bias the analysis.

Second, we have examined the inheritance of previous chromosome 21 DNA markers, as well as the inheritance of new markers from the proximal long arm of chromosome 21 using both extensions of the original four pedigrees, as well as newer 
pedigrees. Lod scores of greater than three can now be generated for $\mathrm{D} 21 \mathrm{~S} 1 / \mathrm{D} 21 \mathrm{~S} 11[\hat{\mathrm{Z}}=3.5$ at $\hat{\theta}-0.16]$. The relative map locations of the new markers are currently being ascertained so that appropriate multipoint lod scores can be calculated. In the initial report, the multipoint lod score curve was extremely broad, and it was, therefore, not possible to determine the location of the FAD gene with respect to D21S1/D21S11 and S21S16. The results of multipoint analyses using additional pedigrees and additional markers may provide a better localization of the FAD gene, but could well be confounded by false recombinant events introduced by the problems noted above. Considerable effort is currently being expended to identify some of the potential confounding factors such as non-paternity, inbreeding, misdiagnosis or inclusion of cases in the same family with identifiably different phenotypes (suggesting two types of $A D$ in the same family), etc.

Third, because of the possibility of non-allelic heterogeneity, we have examined pooled linkage data from eight collaborating centers. To date, the application of appropriate tests [eg. HOMOG, B-test] have failed to provide statistical evidence of linkage heterogeneity. This result is similar to that found by Goate et al. ${ }^{7}$ It is very important to note, however, that failure to detect heterogeneity obviously neither excludes its existence nor proves homogeneity. The most rigorous proof of non-allelic or genetic heterogeneity will be the discovery of other loci on other chromosomes causing FAD in other pedigrees. Consequently, other chromosomal locations are being surveyed for evidence of linked loci by several groups. As of this writing, however, no chromosomal location other than chromosome 21 has been found to show unequivocal evidence of linkage with familial Alzheimer's disease.

\section{Conclusions}

Preliminary data from several different types of epidemiologic studies suggest that at least some cases of $A D$ are the result of an inherited defect in an autosomal gene. At the current time, however, it is not possible either to achieve a definitive estimate of the proportion of $A D$ which results from such a genetic lesion, or to define a phenotype which identifies a genetic form of $A D$.

Recent molecular genetic studies from three independent groups have suggested that the peri-centromeric regions of chromosome 21 may contain a locus for susceptibility to AD. However, two other groups do not confirm these reports. The latter observations have led to the suggestion either that the initial reports are incorrect, or that $\mathrm{AD}$ may be etiologically heterogeneous (multiple genes or genetic and environmental etiologies).
Progress in this field is likely to be slow in the near future because of the presence of several potential confounding factors such as limited availability of large pedigrees with FAD and possible non-allelic heterogeneity, not only between different pedigrees but also even within affected members of the same family.

\section{REFERENCES}

1. Breitner JC, Silverman JS, Mohs RC, et al. Familial aggregation in Alzheimer's disease: comparison of risk among relatives of early and late onset cases and among male and female relatives in successive generations. Neurology 1988; 38: 207-212.

2. Foncin JF, Salmon D, Supino-Viterbo V. Demence Presenile D'Alzheimer Transmise Dans une Famille Etendu. Rev Neurol [Paris] 1985; 141: 194-202.

3. St. George-Hyslop PH, Tanzi RE, Polinsky R, et al. Molecular genetics of autosomal dominant familial Alzheimer's disease. In: Davies P, Finch CE, eds. Current Communications in Molecular Biology. Cold Spring Harbor, NY: Cold Spring Harbor Laboratory (in press).

4. St. George-Hyslop PH, Tanzi RE, Polinsky R, et al. The genetic defect causing familial Alzheimer's disease maps on chromosome 21 . Science 1987; 235: 885-890.

5. Tanzi RE, St. George-Hyslop PH, Haines JL, et al. The beta amyloid gene is not closely linked to the FAD defect. Nature 1988; 331: 528-530.

6. David F, Lucotte G. Co-segregation between D2IS1S11 and the FAD gene in a pedigree. Alz Dis Assoc Disorders 1988; 2: 287.

7. Goate A, Haynes AR, Owen MJ, et al. Predisposing locus for Alzheimer's disease on chromosome 21. Lancet 1989; 1: 352355.

8. Van Broeckhoven C, Van Hul W, Backhoeven $\mathrm{H}$, et al. The familial Alzheimer's disease gene is located close to the centromere of chromosome 21. Am J Hum Gen 1988; 43: 8205.

9. Roses AD, Pericak-Vance MA, Dawson DV, et al. Standard likelihood and sibpair linkage analysis in late-onset Alzheimer's disease. In: Davies P, Finch CE, eds. Current Communications in Molecular Biology. Cold Spring Harbor, NY: Cold Spring Harbor Laboratory (in press).

10. Schellenberg CD, Bird TD, Wijsman EM, et al. Absence of linkage of chromosome 21 q21 markers to familial Alzheimer's disease. Science 1988; 241: 1507-1510.

11. Heyman A, Wilkinson WF, Hurwitz BJ, et al. Alzheimer's disease: genetic aspects and associated clinical disorder. Ann Neurol 1983; 14: 507-515.

12. Farrer LA, O'Sullivan DM, Cupples LA, et al. Assessment of genetic risk of Alzheimer's disease among first-degree relatives. Ann Neurol (in press).

13. Chandra V, Philipose V, Bell PA, et al. Case control study of late onset "probable Alzheimer's disease". Neurology 1987; 37: 1295-1300.

14. Nee LE, Eldridge $R$, Sunderland T, et al. Dementia of the Alzheimer type: clinical and family study of 22 twin pairs. Neurology 1987; 37: 359-363. 